\title{
A dam around the Water Fountain Nebula?
} \section{The dust shell of IRAS16342-3814 spatially resolved with VISIR/VLT}

\author{
T. Verhoelst ${ }^{1,2, \star}$, L. B. F. M. Waters ${ }^{1,4}$, A. Verhoeff ${ }^{4}$, C. Dijkstra ${ }^{3}$, H. Van Winckel ${ }^{1}$, J. W. Pel ${ }^{5}$, and R. F. Peletier ${ }^{5}$ \\ 1 Insituut voor Sterrenkunde, K.U. Leuven, Celestijnenlaan 200D, 3001 Leuven, Belgium \\ e-mail: tijl.verhoelst@ster.kuleuven.be \\ 2 University of Manchester, Jodrell Bank Centre for Astrophysics, Manchester, M13 9PL, UK \\ 3 Department of Physics and Astronomy, University of Missouri, Columbia, MO 65211, USA \\ 4 Astronomical Institute "Anton Pannekoek", University of Amsterdam, Kruislaan 403, 1098 SJ Amsterdam, The Netherlands \\ 5 Kapteyn Astronomical Institute, Landleven 12, 9747 AD Groningen, The Netherlands
}

Received 4 March 2009 / Accepted 25 May 2009

\section{ABSTRACT}

\begin{abstract}
Context. Bipolar morphologies in planetary nebulae $(\mathrm{PNe})$ are believed to be closely linked to binary central stars. Either by collimating a fast stellar wind or by driving a jet via accretion in the central system, dusty torii or stable disks may be crucial ingredients for the shaping of PNe.

Aims. We study the dust distribution in the very young proto-planetary nebula (PPN) IRAS16342-3814, also known as the Water Fountain Nebula, which is known to show strong bipolar characteristics in the shape of two reflection lobes, and high-velocity collimated molecular outlfows.

Methods. We use the new mid-infrared (MIR) instrument VISIR on the Very Large Telescope (VLT) both in imaging and spectroscopy mode at wavelengths from 8 to $13 \mu \mathrm{m}$.

Results. We present the first spatially resolved MIR observations of a dusty evolved star obtained with VISIR and find that the improved spatial resolution contradicts previous claims of an elliptical brightness distribution at the heart of IRAS16342: we find the waist region to be dark even in the MIR. We show that the filling angle of the obscuring dust lane, which is made mostly of amorphous silicates, is very large, possibly even close to a spherically symmetric superwind as seen in OH/IR stars.

Conclusions. We conclude that, in contrast to the multitude of recent dusty-disk detections in post-AGB stars and PNe, IRAS16342 does not show this extreme equatorial density enhancement, at least not on the scale of the dusty environment which lends the object its IR appearance. Rather, it appears that the observed precessing jets are shaping the bipolar nature in the remains of a spherically symmetric AGB superwind.
\end{abstract}

Key words. techniques: high angular resolution - techniques: spectroscopic - stars: AGB and post-AGB - circumstellar matter stars: individual: IRAS16342-3814 - planetary nebulae: general

\section{Introduction}

Two different mechanisms for the shaping of bipolar and multipolar (proto-)planetary nebulae (PNe) are currently under intense investigation (e.g., Meixner et al. 2004). The first is in concept based on the Generalized Interacting Winds model (Balick 1987) and suggests the collimation of the fast, spherical wind of the hot central star by an older, slow, axi-symmetric and possibly warped, AGB (super-)wind (e.g. Icke 2003). The axi-symmetric shaping of the earlier AGB wind may require the central star to be in a binary system.

The other scenario postulates that the lobes are in fact cavities blown in a preceding spherical AGB wind by (precessing) jets (Sahai et al. 2005, 2007). The jets are then most likely the consequence of (re-)accretion of circumstellar material, maybe also in a binary system. This scenario is especially attractive to explain very young PPNe, in which the central star is not yet hot enough to exhibit a strong line-driven wind. These hypotheses are not mutually exclusive.

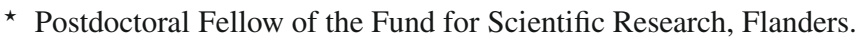

With the advent of powerful mid-IR interferometers, especially MIDI at the VLTI, the type of dust disks which could collimate the fast post-AGB wind have now been observed around binary post-AGB stars (Deroo et al. 2006, 2007), and observations of the dust at the heart of the Ant Nebula (Chesneau et al. 2007) are consistent with a disk interpretation. On the other hand, optical and near-IR observations of some PPNe show evidence for shaping by (precessing) jets (Sahai et al. 2007). The presence of a dusty torus is also suggested in these objects, but it is hitherto unclear whether these tori are similar to the disks observed with MIR interferometry.

IRAS16342-3814, hereafter IRAS16342 is a young PPN. In spite of its extremely red SED, with crystalline silicate features in absorption up to almost $45 \mu \mathrm{m}$ (Dijkstra et al. 2003), optical HST images show a bipolar reflection nebula with a dark equatorial waist (Sahai et al. 1999, hereafter STM99). According to STM99, the lobes are cavities blown by a bipolar molecular jet, observed as high-velocity lines of water (hence the designation "water fountain nebula") and OH (Likkel \& Morris 1988), within the remains of a low mass-loss AGB wind which preceeded the recent superwind phase. Sahai et al. (2005) interpret local density enhancements in the bipolar cavities as due to 
precession of the jet. The dark waist, origin of the bulk of the energy in the SED, is assumed to be an optically thick dusty torus which completely obscures the central star in the line-of-sight to the observer.

Dijkstra et al. (2003), hereafter DKW03, present infrared ISAAC and TIMMI 2 images from 3.8 to $20 \mu \mathrm{m}$, and find a bipolar nature such as in the HST images only at the shortest wavelengths. At longer wavelengths, the source appears elliptical, as was already found by Meixner et al. (1999).

In this paper, we present a new MIR image and an $N$-band spectrum of IRAS16342 obtained with the VISIR instrument on the VLT, both of which resolve the dust structure and oblige us to question our understanding of this (type of) source.

\section{Observations}

The observations presented here were obtained as part of the Dutch GTO on VISIR, the MIR imager and spectrometer installed at the Cassegrain focus of Melipal at the VLT. An image was made in the $\operatorname{SiC}$ filter $\left(\lambda_{\mathrm{c}}=11.85 \mu \mathrm{m}, \Delta \lambda=2.34 \mu \mathrm{m}\right)$ with a 0.127 arcsec PFOV on the night of 20 March 2006, bracketed by 2 PSF observations (HD 146051, M0.5III). Low resolution spectroscopic observations in 4 bands (centered at 8.5, 9.8, 11.4 and $12.2 \mu \mathrm{m}$ ) were made in the night of 17 March 2006 with a $32 \times 0.75 \operatorname{arcsec}$ slit oriented $55.4^{\circ}$ east of north, and a 0.127 arcsec PFOV. The calibrator was again HD 146051. The visual seeing was about 0.60 arcsec for the imaging observations and 0.90 arcsec at the time of the spectroscopy.

For the data reduction, we use the pipeline offered by ESO, version 1.3.7. The synthetic $N$ band spectrum of the calibrator is computed using a MARCS atmosphere model (Gustafsson et al. 1975, and further updates), including $\mathrm{SiO}$ opacity. The stellar parameters are taken from Bordé et al. (2002). The telluric correction of the $N$ band spectra is performed by solving for instrumental efficiency and atmospheric optical depth using the 2 observations of HD 146051. The resulting numbers are then interpolated in airmass to match the conditions at the time of the science observation. As absolute calibration, we scale the VISIR spectrum in such a way that the simulated inband power over the IRAS $12 \mu \mathrm{m}$ filter matches the observed value of $16.2 \mathrm{Jy}$ (see Sect. 3.1 for a discussion on possible slit losses).

\section{Bipolar even in the Mid-IR}

The image deconvolution is performed with both a maximumlikelihood (Richardson 1972; Lucy 1974) and a maximumentropy method (Agmon et al. 1979). Differences between the results of the two methods are minimal. The raw and deconvolved images are shown in Fig. 1.

We observe a double-peaked intensity distribution with a separation of 0.92 arcsec and a position angle (PA) of $66^{\circ}$ east of north. Component W (west) contains about $3 / 4$ of the total flux, component $\mathrm{E}$ (east) the remaining quarter. TIMMI2 images in the $N$ and $Q$ bands observed by DKW03 do not resolve 2 separate emission peaks, but show instead an elliptical shape with a major axis oriented more or less like the separation vector observed at shorter wavelengths. The PA of the object in our VISIR image is compatible with the values found in the optical and near-IR, but the separation we find at $11.85 \mu \mathrm{m}$ is slightly smaller than that at shorter wavelengths. We conclude that the elliptical intensity distribution observed by Meixner et al. (1999) and DWK03 is due to insufficient spatial resolution in their observations.
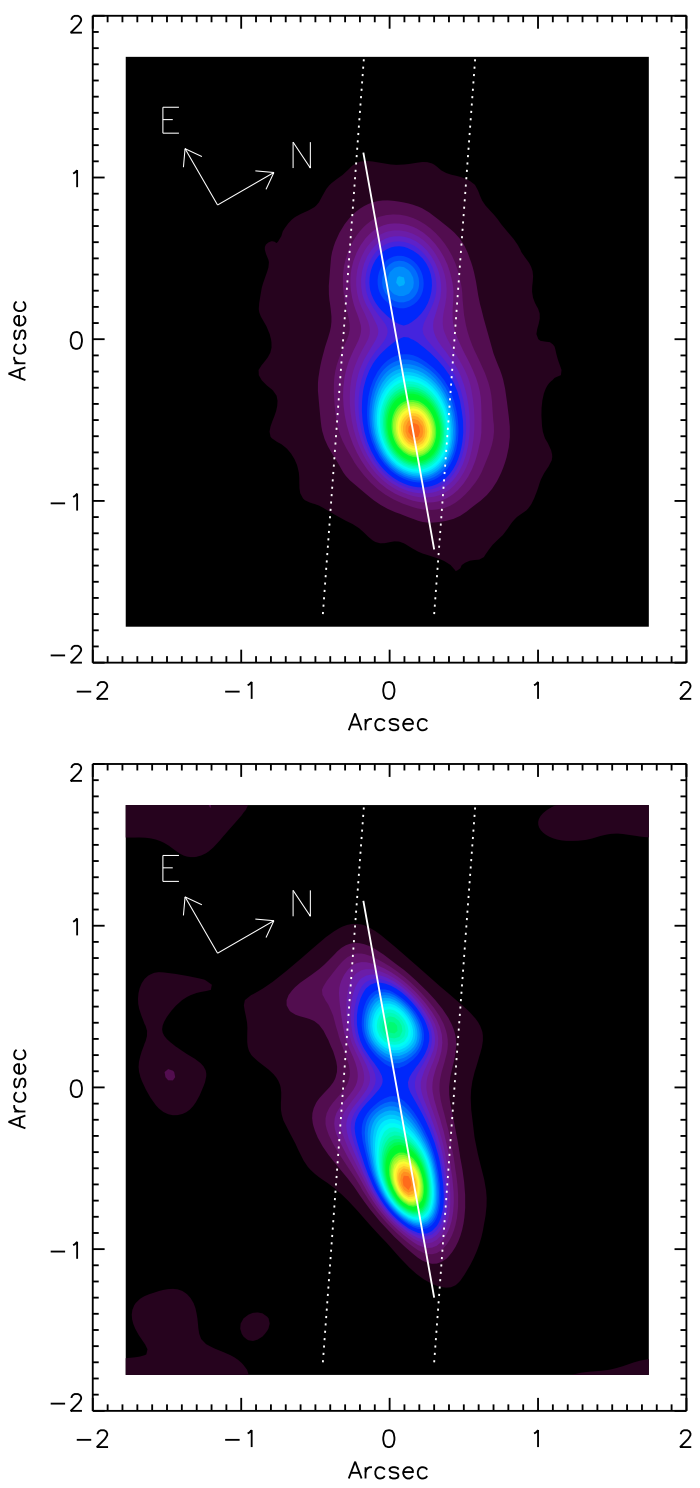

Fig. 1. Upper panel: the raw image of the Water Fountain Nebula in the $\mathrm{SiC}$ filter $(11.85 \mu \mathrm{m})$. The colour scale is linear. The direction of the bipolar optical reflection nebula is also indicated (solid line, from STM99). The dotted lines represent the position and width of the slit used for the spectroscopic observations. Lower panel: the same image but deconvolved using the PSF observation of HD 146051 and with the colour scale following $\mathrm{I}^{(1 / 2)}$. The solid and dotted lines have the same meaning as in the upper panel.

In the deconvolved image, it is apparent that both lobes do not point at a common centre of symmetry. This was also observed by Sahai et al. (1999) in the optical HST images.

Using the central star parameters ${ }^{1}$ of DKW03 $\left(T_{\text {eff }}=2670 \mathrm{~K}\right.$ and $R_{*}=372 R_{\odot}$ ), the distance of $2 \mathrm{kpc}$ and inclination $i=40^{\circ}$ from STM99, and assuming that the dust temperature $T_{\mathrm{d}}$ scales with the distance $r$ from the central star as $T_{\mathrm{d}}=T_{\mathrm{eff}}\left(2 r / R_{*}\right)^{-0.4}$ (e.g. Herman et al. 1986), we find that $T_{\mathrm{d}} \sim 160 \mathrm{~K}$ at $900 \mathrm{AU}$, i.e. the distance between the central star and the approximate center of either lobe. Both numbers are uncertain to at least $20 \%$, but what follows does not depend sensitively on these numbers.

\footnotetext{
1 These parameters correspond to a star at the tip of the AGB, but we can not rule out a more evolved central star. This would imply a slightly higher temperature for the small dust grains, but has no major consequences for the results discussed here.
} 


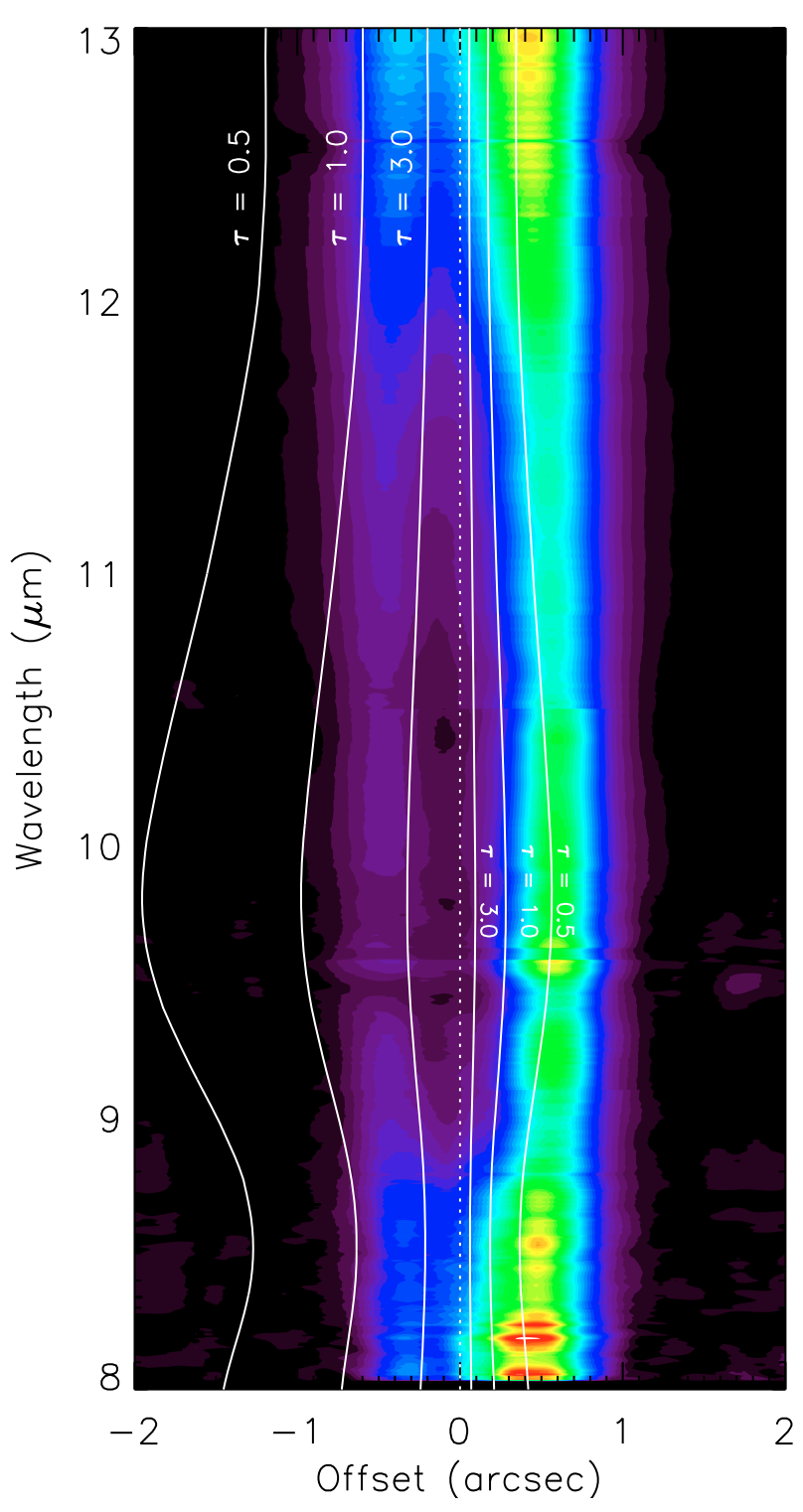

Fig. 2. The reduced and calibrated, but not yet collapsed, spectra (4 bands) pasted together and normalized to the continuum level as derived from a spline fit to the ISO-SWS spectrum (DKW03). The colour scale is linear. Perusal of this figure already reveals that the dark waist reduces the flux level of both lobes around $10 \mu \mathrm{m}$, but more so for the eastern lobe. The solid white lines represent contours of constant opacity following the model presented in Sect. 4.

Using Wien's law, we find a peak of the energy distribution just to the red of the $N$-band. Given the spectral shape of the lobes over the $N$-band presented in Sect. 3.1, we conclude that the $\mathrm{N}$-band image and spectrum are dominated by thermal emission from the dust in the bipolar lobes, which is heated directly by the central star. The material in between, which is obscuring the central star, remains dark even in the MIR, indicating that along the line-of-sight, optical depth $\tau=1$ is reached already in the cold $(T<100 \mathrm{~K})$ outer regions of the dusty environment.

\subsection{The spectra}

The four reduced 2D spectra (at 8.5, 9.8, 11.4 and 12.2 $\mu \mathrm{m}$ ) are shown in Fig. 2, one above the other following increasing wavelength. As shown in Fig. 1, slit losses should at most be of the

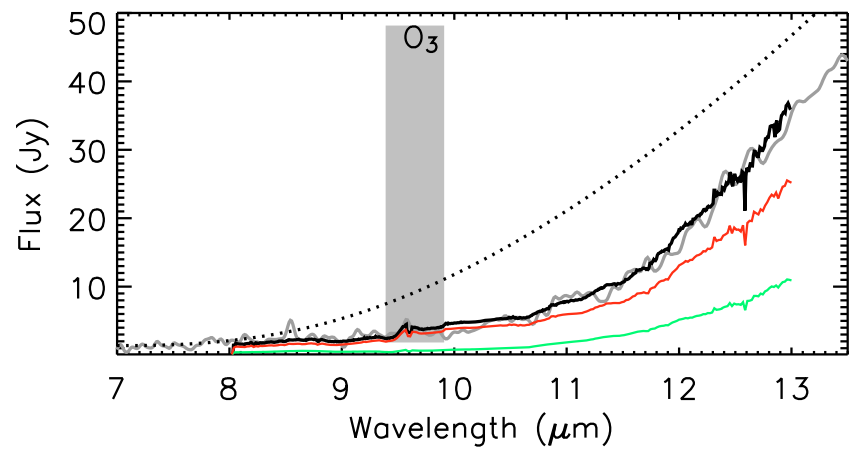

Fig. 3. In black, we show the field-integrated pipeline-extracted spectrum calibrated as discussed in Sect. 2. For comparison, the ISO-SWS spectrum and spline-fit continuum of DKW2003 are shown in grey and dotted lines respectively. Shown in red and green are the separated spectra of the Western and eastern lobes respectively. The grey box indicates the wavelength region of strong atmospheric ozone absorption. The calibration procedure presented in Sect. 2 resulted in reliable data even at these wavelengths.

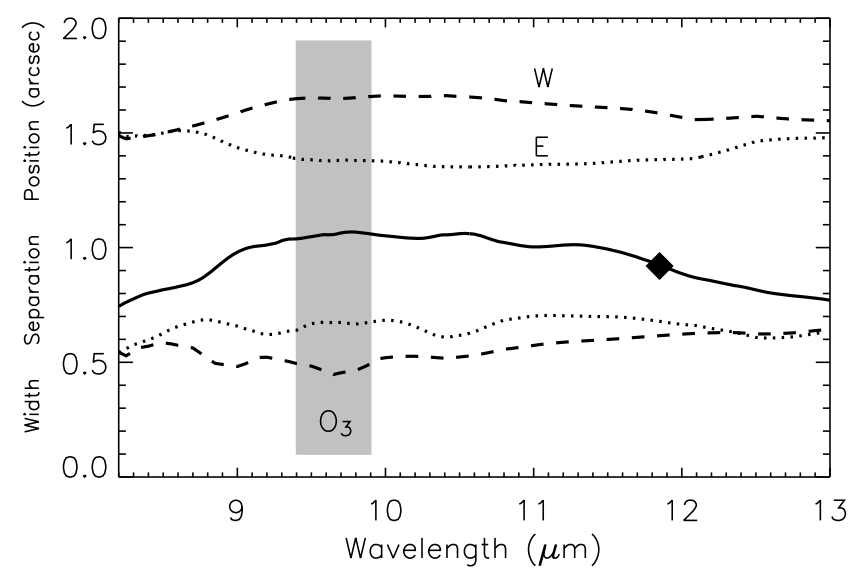

Fig. 4. The dotted and dashed lines represent the position of the E and W lobes respectively, minus the position of their continuum at $8.5 \mu \mathrm{m}$ and offset by 1.5 arcsec for clarity. The solid line represents the separation between component $\mathrm{E}$ and $\mathrm{W}$ as a function of wavelength. The black diamond at $11.85 \mu \mathrm{m}$ indicates the separation measured in the 2D image presented in Sect. 3. The two curves at the bottom show the width of the individual components.

order of a few percent. From this 2D spectrum, we can derive the total field-integrated spectrum, shown as the black solid line in Fig. 3, which is of higher quality than the ISO-SWS spectrum of DKW03 (shown in grey, scaled to match the absolute IRAS flux level) but has the same global shape and no particular spectral features $^{2}$ : in contrast to what is observed at longer wavelengths, we do not find a clear indication of crystalline silicates (e.g. the Forsterite feature at $11.3 \mu \mathrm{m}$ ) within the $N$ band

More interesting is the possibility to separate the eastern and Western lobes in this 2D spectrum. The slit PA of $55.4^{\circ}$ is very close to the object PA of $66^{\circ}$ derived from the VISIR image presented in Sect. 3. To extract position, width and flux levels for the individual spectra, we performed a row-wise fit with a double Gaussian intensity distribution. The 2 lobes can be fully separated through the entire wavelength range.

The resulting wavelength-dependent positions and widths for both lobes are shown in Fig. 4. The wavelength-dependence

2 The absorption line at $12.5-12.6 \mu \mathrm{m}$ is telluric. 


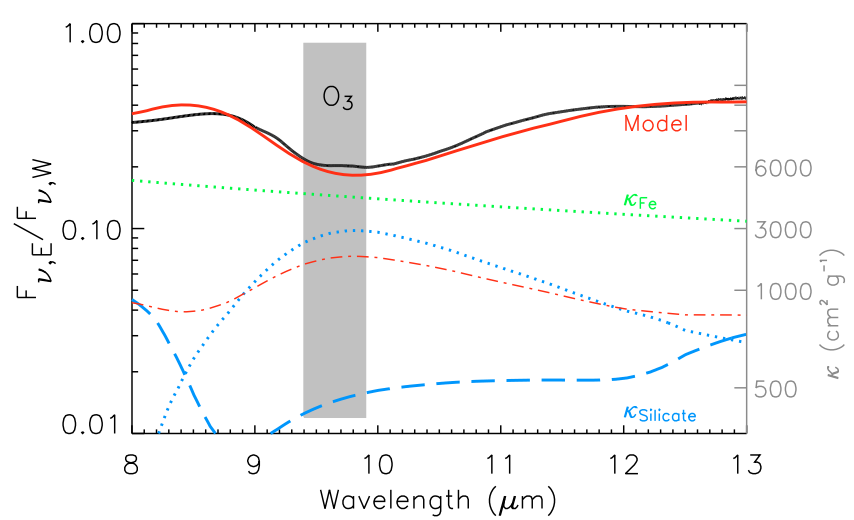

Fig. 5. The observed flux ratio between component $\mathrm{E}$ and $\mathrm{W}$ (black solid line) and the model presented in Sect. 4 (red solid line). Also shown are typical absorption (dotted lines) and scattering (dashed lines) cross sections for iron grains, and for a mix of small and large silicate grains. The scattering by iron grains is negligible at these wavelengths. The red dash-dot line represents the $\kappa(\lambda)$ profile of the best-fit dust composition.

of the separation between both lobes clearly resembles the opacity profile of amorphous silicates, the primary consituent of the dust grains surrounding the central star. The presence of such a wavelength dependence shows that the extinction is not due to a uniform screen of dust (ISM or detached relic of an earlyAGB wind) but instead that, for any given wavelength, the opacity decreases with distance from some point in between both lobes. This is also confirmed by the detection of a varying width in the marginally resolved individual lobes ${ }^{3}$. Since both lobes are affected by the dark waist, we find a disagreement with the model presented by DKW03, in which the flat dusty disk does not result in any obscuration of the Western lobe.

The individual spectra of the eastern and Western lobes are presented in Fig. 3, and in Fig. 5 we display the ratio between both. The individual spectra show that both lobes emit an equally cold spectrum, and both exhibit the silicate feature in absorption. This is again in disagreement with the low filling angle of the model by DKW03 which predicts a dust emission spectrum for the Western lobe.

The flux ratio shows that the eastern lobe suffers stronger attenuation than its Western counterpart. Again, the major feature is that of amorphous silicates. A proper identification of the type of silicates would also require the observation of the $18 \mu \mathrm{m}$ band. The flux ratio $F_{v, \mathrm{E}} / F_{\nu, \mathrm{W}} \sim 0.4$ (instead of unity) at the "continuum" wavelengths of 8.5 and $13 \mu \mathrm{m}$ indicates achromatic attenuation, which is most likely due to metallic Fe, a crucial ingredient in the SED modelling of OH/IR stars and obscured RSG (Kemper et al. 2002; Harwit et al. 2001). The observed absorption towards $8 \mu \mathrm{m}$ can be attributed to amorphous silicates, but only through scattering by fairly large grains. This suggests that a fraction of the obscuring dust is contained in micron-sized grains, which was already suggested for the dark waist by DKW03, albeit based on a geometrically inadequate model as discussed above.

Having found that the obscuring dust structure must have a significant filling angle, we try to quantify this in the following section.

3 The standard star observations show a width of $0.27 \pm 0.03$ arcsec.

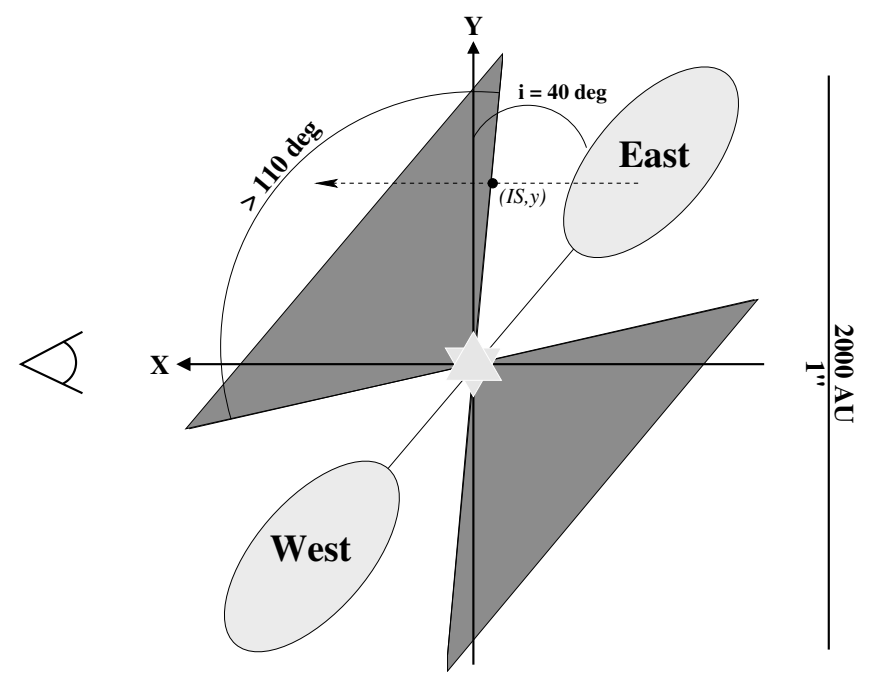

Fig. 6. The toy model used to estimate the filling angle of the obscuring dust structure. The density in the dust structure drops with $r^{2}$, where $r$ is the distance to the central star. The inclination is taken from SAH99. $(I S, y)$ is the intersection between a line-of-sight and the inner edge of the dust structure. The lobes emit thermal radiation at $160 \mathrm{~K}$.

\section{Interpretation: filling angle}

Located at a similar distance as many known post-AGB binaries, i.e. a few kpc, IRAS16342 appears much larger in the midIR than the stable dusty disks around those stars: 1 arcsec vs. 40 mas respectively. Equivalently, the observed strong extinction toward the eastern lobe requires a significant dust column at least $500 \mathrm{AU}$ above the midplane, and this is not possible in a stable Keplerian dust disk with a reasonable mass. The dust must therefore have some radial velocity.

With a simple geometrical model (see Fig. 6), which is not intended to be a detailed representation of the source with all its peculiar characteristics, it is possible to get an estimate of the filling angle and dust mass of the obscuring dust structure from the spatially resolved $N$-band spectrum. The mid-IR data presented here have as an advantage over the optical and near-IR data presented hitherto that the modelling is much less sensitive to scattering. Including scattering in the analysis would add a new level of complexity (e.g. the central source spectrum, the scattering properties of the dust at short wavelengths, the scattering phase function), and is not required to constrain the basic structural parameters. We leave the construction of a full "colour" image to a future paper.

In the assumption of an outflow with a biconical cavity, the optical depth $\tau$ along a line-of-sight at a distance $y$ from the center is calculated as:

$$
\begin{aligned}
\tau(y, \lambda)_{E, W} & =\int_{\mathrm{IS}}^{\infty} \frac{\rho_{0} \kappa(\lambda)}{x^{2}+y^{2}} \mathrm{~d} x \\
& =\frac{\rho_{0} \kappa(\lambda)}{y^{2}}[\arctan (x / y)]_{y \tan \left(\frac{\pi}{2}-\frac{\theta}{2} \pm i\right)}^{\infty} \\
& =\frac{\rho_{0} \kappa(\lambda)}{y^{2}}\left(\frac{\theta}{2} \pm i\right) .
\end{aligned}
$$

It depends on (1) the filling angle $\theta$ of the dust structure, (2) the dust density $\rho_{0}$ at unit radius ${ }^{4}$, and (3) the dust mass fraction

\footnotetext{
$\overline{4}$ The density follows a r ${ }^{-2}$ distribution, with a $15 \mathrm{~km} \mathrm{~s}^{-1}$ outflow velocity.
} 
caught in small and large silicate grains ${ }^{5}(0.1$ and $5 \mu \mathrm{m})$, and in metallic iron, implicit in the wavelength-dependent cross section $\kappa$. The "background" radiation by the lobes is assumed to be thermal emission by small dust grains at $160 \mathrm{~K}$, as derived in Sect. 3. Whether these small dust grains fill the cavities or form the boundary with the denser dust structure can not be derived from our current observations. We do not assume an actual density or temperature distribution for the individual lobes: the lobes appear marginally resolved in our observations, but insufficiently so to derive such a distribution. The comparison between model and observations is therefor done at the "nominal" distances of $y_{0}=0.45 \operatorname{arcsec}(900 \mathrm{AU})$ from the center of the object. We determine the unknowns by requiring reproduction of (1) the very red SED up to $20 \mu \mathrm{m}$ with total obscuration of the central star and hot inner dust (from the optical to the mid-IR), (2) the wavelength-dependent flux ratio between both lobes; and (3) the moderate observed extinction towards the Western lobe $\left(\tau_{10 \mu \mathrm{m}} \sim 0.5\right.$, derived from the depth of the silicate absorption feature in the Western spectrum). The wavelength dependence of the dust opacity is mostly constrained by (2). The dust density is determined by (1) and (3).

We find that a filling angle of at least $110 \mathrm{deg}$ is required to produce observable extinction towards the Western lobe. The best fit is obtained with a 145 deg filling angle. With a dust species mass ratio of $\frac{\rho_{\text {silicates }}}{\rho_{\mathrm{Fe}}}=5.5$, a silicate size dust mass ratio of $\frac{\rho_{0.1 \mu \mathrm{m}}}{\rho_{5.0 \mu \mathrm{m}}}=1.5$, and a mass-loss rate of $\dot{M} \sim 1 \times 10^{-3} M_{\odot} \mathrm{yr}^{-1}$, we find good agreement with the $N$-band observations (Fig. 5) and lack of flux at shorter wavelengths (toward the central star, our model predicts $\tau \geq 100$ up to $30 \mu \mathrm{m}$ ). However, we can not exclude an even larger filling angle.

\section{Discussion and conclusions}

We find that, in spite of previous classifications as an elliptical MIR source, IRAS16342 in fact appears as a bipolar source up to at least $13 \mu \mathrm{m}$, if studied with sufficient angular resolution. Almost all of the $N$-band flux originates in these 2 lobes, with an apparant temperature of roughly $160 \mathrm{~K}$. The dusty "waist" region remains dark even at these wavelengths. From the varying extinction in front of both lobes as a function of wavelength, we derive that the dust structure has a large filling angle $\left(\sim 145^{\circ}\right)$, much larger than the stable Keplerian dust disks recently seen around other binary post-AGB stars (typically $40^{\circ}$, de Ruyter et al. 2006; Deroo et al. 2007), and this in spite of the strong indications that IRAS16342 also has a binary central system. Instead, it resembles more the spherically symmetric superwind of an $\mathrm{OH} / \mathrm{IR}$ star, but with cavities blown by jets. Unfortunately, the dam around this fountain is too high to see the engine driving the bipolar jets. It will be interesting to see whether all members of the class of Water Fountain Nebulae display this kind of dust structure.

Acknowledgements. The authors would like to thank the anonymous referee for many valuable comments, and E. Lagadec for a careful reading of the manuscript.

\section{References}

Agmon, N., Alhassid, Y., \& Levine, R. D. 1979, J. Comput. Phys., 30, 250 Balick, B. 1987, AJ, 94, 671

Bordé, P., Coudé du Foresto, V., Chagnon, G., et al. 2002, A\&A, 393, 183

Chesneau, O., Lykou, F., Balick, B., et al. 2007, A\&A, 473, L29

de Ruyter, S., van Winckel, H., Maas, T., et al. 2006, A\&A, 448, 641

Deroo, P., van Winckel, H., Min, M., et al. 2006, A\&A, 450, 181

Deroo, P., Acke, B., Verhoelst, T., et al. 2007, A\&A, 474, L45

Dijkstra, C., Waters, L. B. F. M., Kemper, F., et al. 2003, A\&A, 399, 1037

Gustafsson, B., Bell, R. A., Eriksson, K., \& Nordlund, A. 1975, A\&A, 42, 407

Harwit, M., Malfait, K., Decin, L., et al. 2001, ApJ, 557, 844

Herman, J., Burger, J. H., \& Penninx, W. H. 1986, A\&A, 167, 247

Icke, V. 2003, A\&A, 405, L11

Kemper, F., de Koter, A., Waters, L. B. F. M., Bouwman, J., \& Tielens, A. G. G. M. 2002, A\&A, 384, 585

Likkel, L., \& Morris, M. 1988, ApJ, 329, 914

Lucy, L. B. 1974, AJ, 79, 745

Meixner, M., Ueta, T., Dayal, A., et al. 1999, ApJS, 122, 221

Meixner, M., Kastner, J. H., Balick, B., \& Soker, N., 2004, ASP Conf. Ser. 313, Asymmetrical planetary nebulae III: proceedings of a meeting held at Mt. Rainier, Washington, 28 July-1 August 2003

Richardson, W. H. 1972, Opt. Soc. Am. J. A, 62, 55

Sahai, R., Te Lintel Hekkert, P., Morris, M., Zijlstra, A., \& Likkel, L. 1999, ApJ, 514, L115

Sahai, R., Le Mignant, D., Sánchez Contreras, C., Campbell, R. D., \& Chaffee, F. H. 2005, ApJ, 622, L53

Sahai, R., Sánchez Contreras, C., Morris, M., et al. 2007, ApJ, 658, 410

\footnotetext{
5 We use only these 2 discrete sizes since there is no reason to believe that a classical power law size distribution is valid for this dust structure.
} 